\title{
Restauração estética de dentes conóides associada ao recontorno gengival em paciente jovem: relato de caso
}

\author{
Bruno Fernandes OLIVEIRA'; Juliano Gonçalves \\ MIGUEL'; Ana Paula Rodrigues MAGALHÃES ${ }^{3}$ \\ 1 - Graduando em Odontologia pela Universidade Paulista (FO/UNIP), Goiânia-GO, Brasil; 2 - Mestre em Ciências da Saúde pela \\ Faculdade de Medicina (FO/UFG), Professor de Periodontia no Centro Universitário Alfredo Nasser, Aparecida de Goiânia-GO, \\ Brasil; 3 - Doutora em Dentística Restauradora pela Universidade de São Paulo (FOB/USP), Professora titular de Dentística \\ Restauradora na Universidade Paulista, Goiânia-GO, Brasil.
}

\section{Resumo}

A busca por tratamentos dentários por razões estéticas tem aumentado muito devido ao grande impacto do sorriso na autoestima e bem-estar dos pacientes em seu convívio social. No planejamento estético reabilitador critérios dento-faciais, labiais, periodontais e pessoais devem ser levados em consideração na indicação de qualquer tratamento. O objetivo deste artigo é relatar um caso de sorriso gengival e dentes conóides em paciente jovem, tratada com uma abordagem minimamente invasiva. No exame físico foi possível observar o sorriso gengival causado pela erupção passiva alterada e microdontia isolada nos incisivos laterais superiores, o que prejudicava a harmonia do sorriso. Foram propostos alguns planos de tratamento e diante do custo-benefício foram realizados aumento de coroa clínica estético sem retalho ("flapless") dos incisivos centrais e laterais, e pré molares do lado direito superior, associada a clareamento dental caseiro supervisionado e restaurações diretas em resina composta sem preparo prévio da estrutura dentária. As restaurações foram guiadas por uma matriz de silicone obtida imediatamente e desgastada no tamanho final planejado com broca, sem a necessidade de enceramento diagnóstico. O resultado obtido com o tratamento proposto melhorou as proporções dento-faciais e gengivais, solucionando a queixa principal da paciente com preservação de estrutura dental sadia, restabelecendo estética e mantendo a função de forma adequada.

PALAVRAS-CHAVE: Estética dentária; Resinas Compostas; Periodontia; Cirurgia Plástica.

AUTOR PARA CORRESPONDÊNCIA

Ana Paula Rodrigues Magalhães Associação Brasileira de Odontologia - Seção de Goiás

Av. Itália, 1184 - Jardim Europa, Goiânia - GO, CEP: 74325-110

E-mail: anapaulardm@gmail.com 


\section{Introdução}

Um sorriso harmônico tem sido o objetivo da maioria dos pacientes em consultórios odontológicos e está relacionado com a autoestima e autoaceitação desses na sociedade ${ }^{1-5}$. As características buscadas para obtenção desse sorriso estão relacionadas à proporção, simetria e harmonia dos dentes no segmento maxilo-mandibular que se projetam com a geometria da face $e^{4,6,7}$. Discrepâncias estéticas na região anterossuperior relacionadas à cor, proporção, forma, textura, tamanho e posição dos dentes são as principais queixas dos pacientes, bem como anomalias de desenvolvimento, como a microdontia, e o sorriso gengival, que os faz buscar um tratamento estético ${ }^{1,4,5,8,9}$.

A microdontia é uma alteração dentária do desenvolvimento relacionada ao tamanho e forma dos dentes e pode ser classificada em isolada e generalizada verdadeira absoluta, sendo essa última incomum ${ }^{10-12}$. Em uma dentição normal esta alteração de modo isolado acomete um ou mais dentes, frequentemente terceiros molares e incisivos laterais superiores permanentes, sendo observada clinicamente com uma coroa em forma de cone tendo seu tamanho reduzido no sentido mésio-distal e convergência no sentido cérvico-incisal refletindo no aparecimento de diastemas. Radiograficamente, apresentam raízes de comprimento normal, e observa-se uma predileção pelo sexo feminino $0^{1,4,5,7,8,10-16}$, com sua taxa de prevalência variando de $0,8 \%$ a $8,4 \%$ da população ${ }^{12}$.

Modalidades terapêuticas têm sido relatadas na literatura para o tratamento estético de dentes conóides a partir de técnicas mais invasivas e de alto custo como a confecção de coroas unitárias e facetas de porcelana, e técnicas de mínima intervenção ${ }^{8}$. Atualmente, os laminados cerâmicos tornaram-se outra possibilidade, visto que o preparo da estrutura dentária é mínimo, porém oneroso, fazendo com que grande parte da população não tenha acesso a esses procedimentos ${ }^{4,15}$. As restaurações adesivas diretas com resinas compostas permitem um procedimento 
extremamente conservador, minimamente invasivo ou mesmo sem desgastes da estrutura dentária, com uma reanatomização rápida e de custo relativamente baixo, sendo esta modalidade uma excelente alternativa para tratamentos estéticos ${ }^{3,4,7,8,10}$.

A harmonia do sorriso torna-se esteticamente agradável quando há um equilíbrio proporcional entre dentes, gengiva, lábios e uma limitação de até 3 milímetros de exposição gengival na movimentação dinâmica dos lábios ${ }^{17-19}$. A exposição gengival excessiva ou sorriso gengival é uma preocupação estética significativa na população, com dados de prevalência variando de $10,5-29 \%{ }^{20}$ afetando comumente as mulheres ${ }^{19,20}$. Os fatores etiológicos que originam o sorriso gengival frequentemente observados são hiperatividade dos lábios, erupção passiva alterada, lábio superior curto, extrusão dento alveolar, crescimento acentuado vertical da maxila, hiperplasia gengiva ou mistos ${ }^{6,17-23}$. O diagnóstico assertivo auxilia o profissional a propor aos pacientes a terapêutica ideal, com abordagens cirúrgicas, como ortognática, reposicionamento de lábios, gengivoplastia e/ou gengivectomia e osteotomia; ou não cirúrgicas como aparelho ortodôntico e injeção de toxina botulínica ${ }^{6,17-20,22-24}$.

Com o objetivo de atender às necessidades do paciente, o tratamento ideal frequentemente é multidisciplinar para a resolução de diversos casos clínicos. A partir do desenvolvimento de técnicas e materiais restauradores, opções de tratamento estético na região anterossuperior com resina composta associada à gengivoplastia tem sido relatadas em pacientes jovens, preconizando uma filosofia minimamente invasiva, preservando ao máximo estrutura dentária sadia ${ }^{5,7}$. Portanto, o objetivo deste trabalho é relatar um caso de sorriso gengival e dentes conóides em paciente jovem tratada com uma abordagem minimamente invasiva.

\section{Relato de caso}

Uma paciente jovem de 22 anos de idade, melanoderma, do sexo feminino, compareceu à clínica odontológica relatando 
insatisfação com a estética de seu sorriso; estimulada pela aparência dos incisivos laterais superiores que se apresentavam fora dos padrões de forma e tamanho, em virtude de uma alteração dentária do desenvolvimento e uma discreta faixa de tecido gengival quando sorria (Figura 1). Segundo a American Society of Anesthesiologists (ASA) a classificação do estado físico foi ASA I, considerando uma paciente saudável, sem anormalidades fisiológicas, físicas ou psicológicas ${ }^{25}$.

Por meio do exame clínico, foi possível chegar a um diagnóstico de microdontia isolada em incisivos laterais, também conhecida como dentes conóides, e sorriso gengival em virtude da erupção passiva alterada (Figuras 2-4). Foi realizada a sondagem periodontal, utilizando uma sonda do tipo Carolina do Norte, com o intuito de localizar a junção cemento-esmalte (JCE), local este onde será posicionada a futura margem gengival (MG). Além disso, observou-se ausência de sangramento à sondagem,

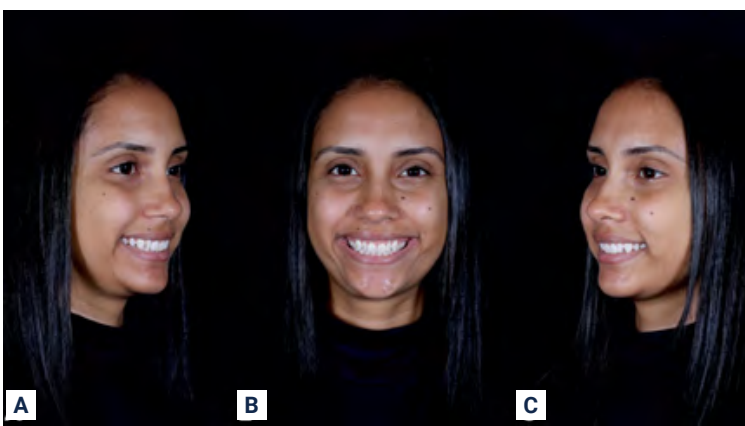

FIGURA 1 - Fotografia inicial da paciente mostrando a discrepância da harmonia do sorriso. (A) Vista lateral direita do sorriso; (B) Vista frontal; (C) Vista lateral esquerda.

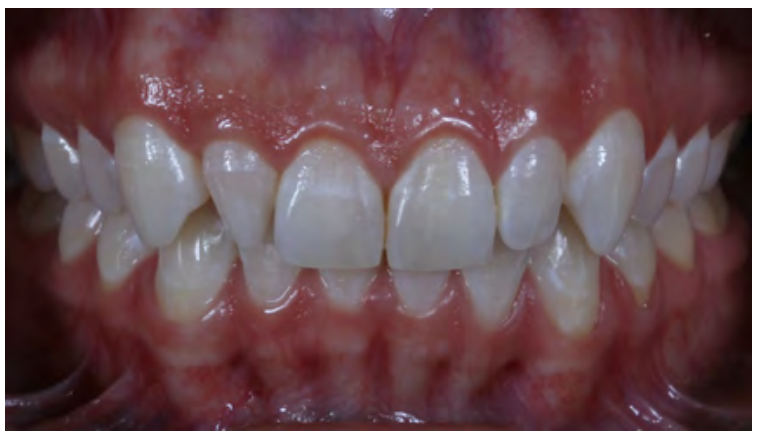

FIGURA 3 - Fotografia intraoral com uso de afastador labial mostrando os dentes conóides e discrepâncias gengivais.



FIGURA 2 - Aspecto inicial do sorriso, mostrando a alteração do desenvolvimento de forma e tamanho, e sorriso gengival, queixa principal da paciente.

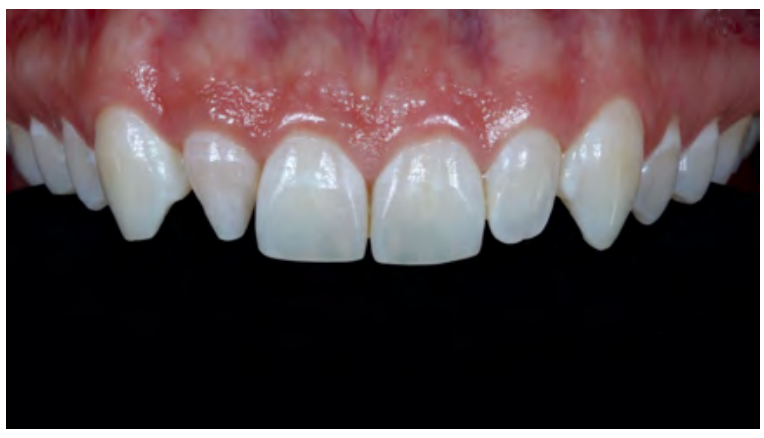

FIGURA 4 - Vista frontal com fundo escuro mostrando o aspecto dos dentes conóides e o periodonto. 
informação que, adicionada aos aspectos macroscópicos da gengiva e à ausência de biofilme bacteriano visível, descartaram a possível presença de edema por inflamação. Foi possível, também, identificar na região anterossuperior coroas clínicas curtas, confirmadas com exames radiográficos que demonstraram ausência de alterações radiculares e comprimento normal dos incisivos laterais (Figura 5).

A partir disso, foi realizado um planejamento digital do sorriso (Figura 6) e foram propostos alguns planos de tratamento à paciente. Um aumento de coroa clínica estético sem elevação de retalho ("flapless") dos incisivos centrais e laterais, e pré-molares do lado direito superiores, conforme protocolo descrito por Carvalho et al. ${ }^{21}$ (2010), seguindo como referência aspectos estéticos e biológicos seria indicado em todas as situações. Complementar à cirurgia, e em busca de atender a queixa principal da paciente, a realização de reanatomização dos incisivos laterais e aumento incisal dos incisivos centrais superiores por meio de restaurações diretas em resina composta ou indiretas por meio de laminados cerâmicos, buscando uma proporção mais adequada entre os dentes, com harmonia e simetria com o periodonto. Após apresentação das vantagens e desvantagens de cada procedimento, além de uma avaliação de custos, a paciente decidiu por fazer a cirurgia periodontal e as restaurações de resina composta.

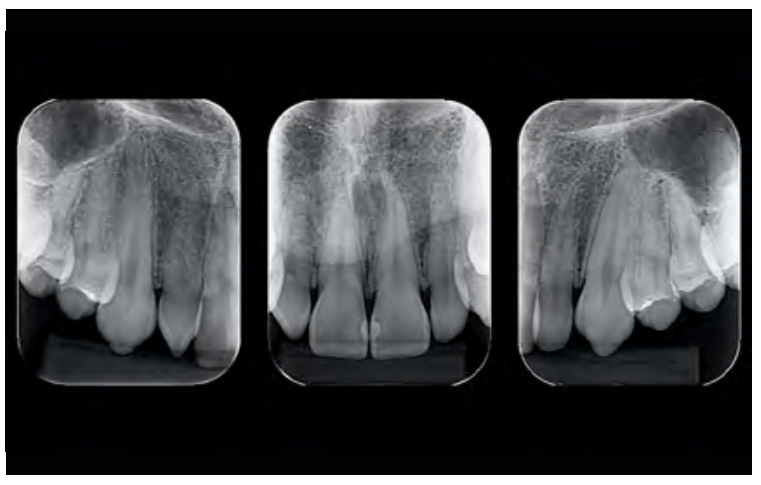

FIGURA 5 - Radiografias periapicais da região anterossuperior com coroas clínicas dos incisivos laterais curtas e respectivas raízes com aspecto de normalidade, compatível com microdontia isolada.

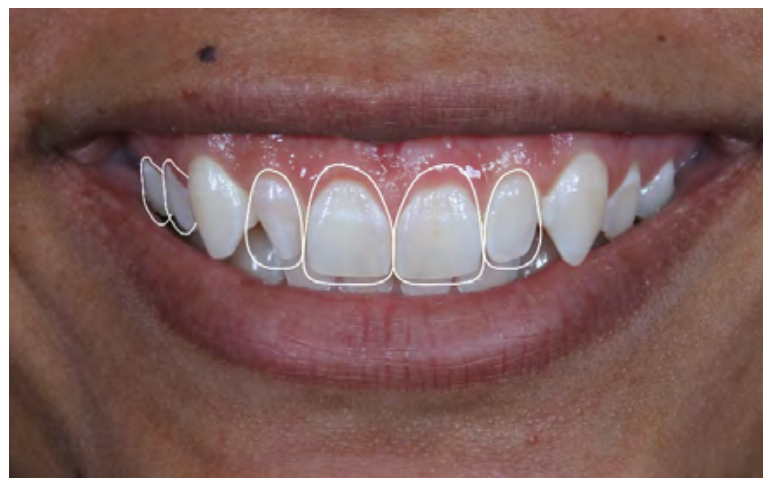

FIGURA 6 - Planejamento digital do sorriso da paciente. 
Previamente ao procedimento cirúrgico, a paciente foi orientada a realizar bochechos com digluconato de clorexidina a $0,12 \%$, durante 30 segundos. Como técnica anestésica para controle durante e conforto no pós-operatório imediato, utilizou-se de mepivacaína 2\% com epinefrina 1:100.000 (Mepiadre, DFL, Rio de Janeiro, Brasil), realizou-se técnicas de bloqueio do nervo alveolar médio, nervo alveolar anterior, complementada por técnica infiltrava local supraperiosteal no fundo de vestíbulo e intrapapilar dos dentes $11,12,14,15,21$ e 22.

Posteriormente, o posicionamento da incisão foi estabelecido na JCE após sondagem e marcação de pontos de orientação, visto que os dentes não apresentavam restaurações, conforme o exame clínico-radiográfico. Com uma lâmina de bisturi 15C (Swann Morton, Sheffield, Reino Unido) foi realizada uma incisão em bisel interno com uma angulação de 45ำ em direção a JCE (Figura 7A). Após a incisão, foi removido o "colar" de tecido excisado com auxílio de uma cureta do tipo Gracey 5/6 (Hu-Friedy, Rio de Janeiro, Brasil) (Figura 7B) e um refinamento da margem gengival com uma micro-tesoura castroviejo curva (Schwert, Oberflacht, Alemanha) (Figura 7C). Isso proporcionou um melhor contorno da margem gengival e um alinhamento da altura dos zênites gengivais dos incisivos centrais ao mesmo nível dos caninos, e dos incisivos laterais levemente mais coronais, conforme contorno estético definido pelo planejamento cirúrgico.

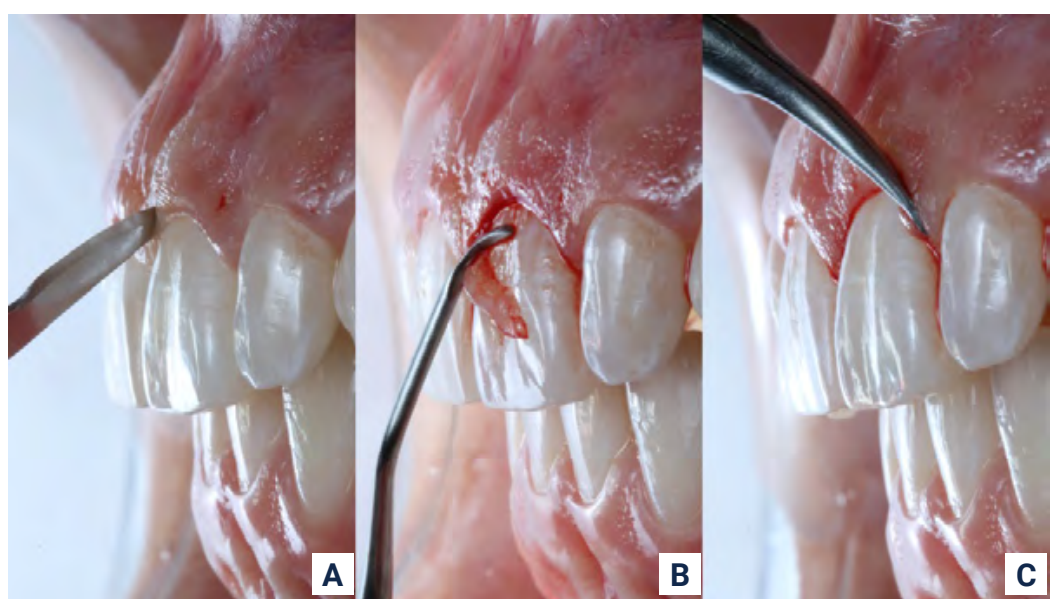

FIGURA 7 - Cirurgia de aumento de coroa clínica estético sem retalho. (A) Incisão em bisel interno no nível JCE; (B) Remoção do tecido excisado com cureta; (C) Regularização da margem gengival e alinhamento dos zênites gengivais com microtesoura. 
Após esta etapa, realizou-se uma sondagem da JCE, definida como nova posição da margem gengival, até a crista óssea alveolar (COA) (Figura 8A). A distância entre MG e COA, é mensurada em todos os dentes após o recontorno, e sempre que menor que $3 \mathrm{~mm}$, realiza-se osteotomia com micro-cinzéis via sulco gengival, sem elevação de retalhos (Figura 8B), conforme determinado pela técnica cirúrgica utilizada, até o estabelecimento de uma nova distância biológica nos parâmetros definidos em trabalhos clássicos ${ }^{26}$. Por conseguinte, deve-se realizar uma nova sondagem, confirmando a profundidade de sondagem e a acomodação dos tecidos gengivais (Figura 8C). A Figura 9 mostra o resultado imediato após a cirurgia periodontal, sem a necessidade de realização de suturas.

A paciente foi orientada quanto ao uso de medicações analgésicas, anti-inflamatórias e controle de biofilme. Para a primeira foi prescrito dipirona sódica em $500 \mathrm{mg}$, utilizada para controle da dor quando presente, por no máximo 4 vezes ao dia. Já a segunda, seria utilizada independente da sintomatologia, nimesulida $100 \mathrm{mg}$, administrada 2 vezes ao dia durante 3 dias, podendo estender por até 5 dias. Por fim, a última, seria por meio de bochechos de digluconato de clorexidina a $0,12 \%$, com duração de 30 segundos, 2 vezes ao dia, durante 10 dias.

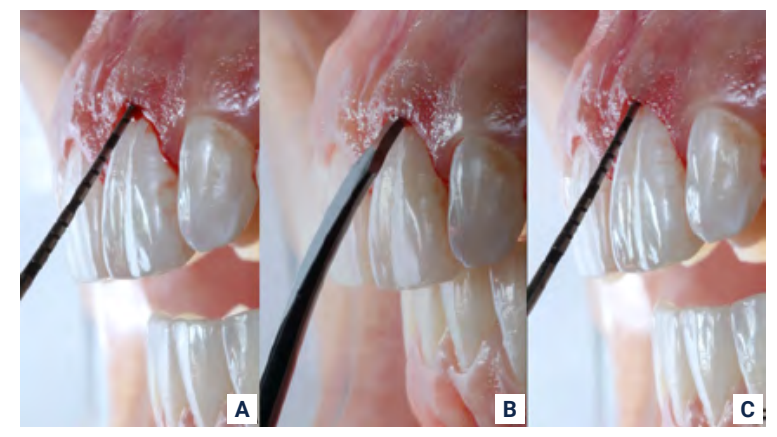

FIGURA 8 - Cirurgia de aumento de coroa clínica estético sem retalho. (A) Sondagem óssea da JCE à COA; (B) Osteotomia via sulco gengival com micro-cinzel sem elevação de retalhos; (C) Profundidade de sondagem final de $3 \mathrm{~mm}$ compatível para acomodação dos tecidos gengivais.

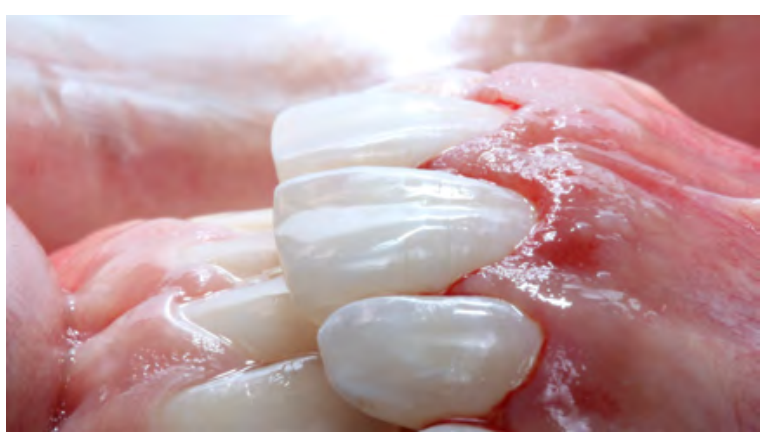

FIGURA 9 - Vista lateral após o término do tratamento cirúrgico periodontal sem necessidade de suturas. 
Após 60 dias da cirurgia periodontal, tempo este necessário para estabilidade dos tecidos periodontais e estabilidade dimensional da margem gengival, a paciente foi reavaliada. Confirmada a cicatrização dentro dos parâmetros estéticos e biológicos, foi realizado clareamento caseiro supervisionado com gel de peróxido de carbamida 20\% (Opalescence, Ultradent, SP-Brasil), durante noventa minutos/dia, por 21 dias.

Para a realização das restaurações de resina composta, foi realizada uma moldagem prévia com silicone de condensação denso (Optosil Comfort Putty/Kulzer, São Paulo, Brasil), adaptando o material nos dentes anterossuperiores, sem o uso de moldeiras de estoque. Após a presa desse material e a aquisição do molde, foi recortada a face vestibular do mesmo com lâmina de bisturi nº 12 (Solidor, Lamedid, Barueri, SP, Brasil) com cautela, a fim de preservar a borda incisal de todos os dentes e manter a face palatina (Figura 10A). A morfologia e tamanho pretendidos dos dentes 11, 12, 21 e 22 foram delimitados com lapiseira no molde (Figura 10B) e, posteriormente, foram cortados com uma ponta diamantada de granulação extrafina 4137 FF (KG Sorensen, São Paulo, Brasil) em alta rotação, com intuito de alcançar o formato planejado dos elementos (Figuras 10C e D), de maneira que a concha palatina em resina composta pudesse ser confeccionada a partir dessa guia de silicone, sem a necessidade de enceramento diagnóstico (Figuras 11A e B).

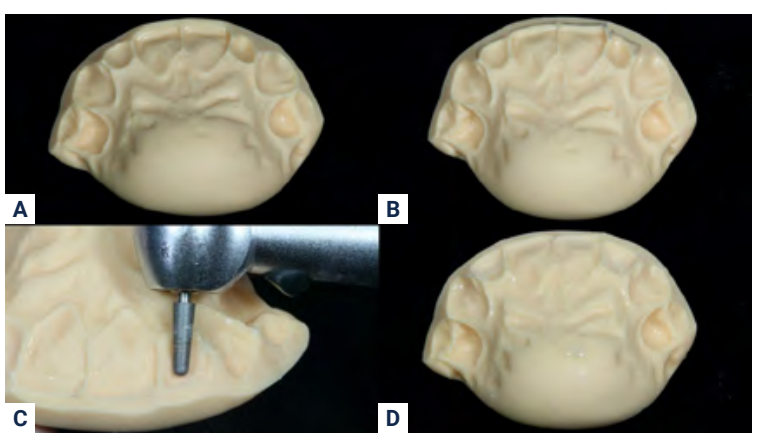

FIGURA 10 • Confecção da guia de silicone para a realização das restaurações em resina composta. (A) Molde em silicone de condensação após o recorte da face vestibular; (B) Delimitação com lapiseira da morfologia e tamanho dos incisivos planejados;

(C) Recorte com ponta diamantada nos limites estabelecidos;

(D) Matriz finalizada para realização das restaurações.

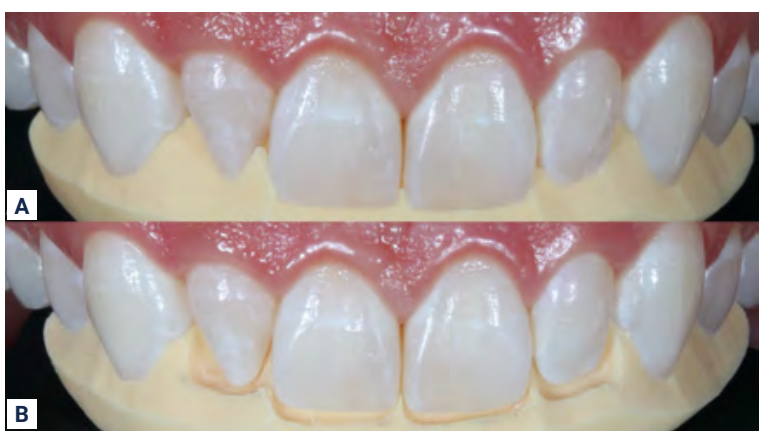

FIGURA $11 \cdot$ (A) Guia de silicone posicionada na cavidade bucal previamente ao recorte da morfologia e tamanho pretendidos dos incisivos; (B) Matriz em posição conforme o planejamento estabelecido. 
Após esta etapa, foi realizada profilaxia com pedra pomes eágua e seleção das cores da resina composta a serem utilizadas. Para a seleção de cor, quatro pequenos incrementos de resinas de diferentes cores e opacidades foram posicionados sobre a superfície dentária, fotopolimerizados com LED (Valo Cordless, Ultradent, South Jordan, UT, Estados Unidos) durante 20s e observados com umidade e sem a luz do foco (Figura 12). Em seguida, seguiu-se com a proteção dos dentes adjacentes com fita de teflon, condicionamento dos dentes a serem restaurados com ácido fosfórico a $37 \%$ (Condac 37, FGM, Joinville, SC, Brasil) por 30 s em esmalte vestibular e palatino, lavagem com spray de água e ar pelo dobro do tempo de condicionamento e secagem. Foi realizado isolamento relativo do campo operatório e aplicação do sistema adesivo universal (Single Bond Universal 3M ESPE, Saint Paul, MN, Estados Unidos) com fricção ativa por 20s, leve jato de ar por aproximadamente 5 s e fotopolimerização por 10 s.

Subsequentemente, foi inserido na guia de silicone um incremento de resina composta em espessura fina na cor WE (Filtek Z350 XT, 3M ESPE, Saint Paul, MN, Estados Unidos) para reconstruir o esmalte palatino, adaptando-a com o auxílio de espátulas específicas de resina e pincel de pelo de marta (Cosmedent nํ 3, Chicago, IL, Estados Unidos) (Figura 13A).

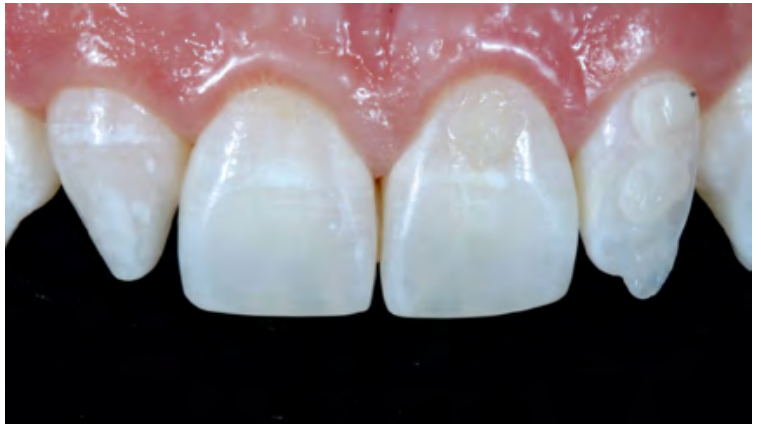

FIGURA 12 - Seleção de cores a partir de pequenos incrementos de resinas compostas com diferentes cores e opacidades fotopolimerizadas sobre a superfície dentária.

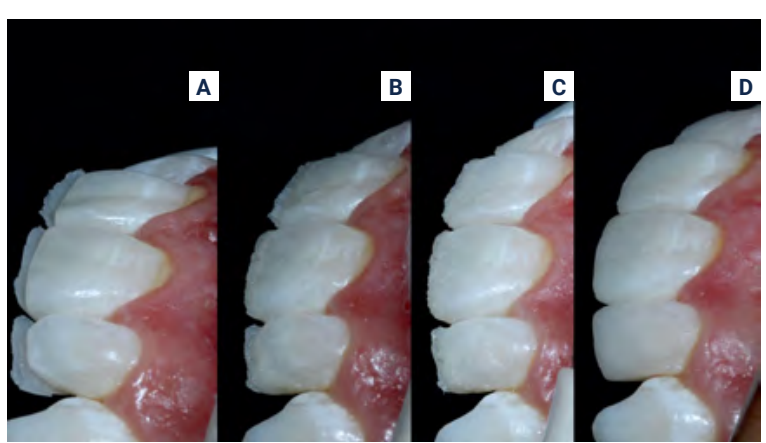

FIGURA 13 - Realização das restaurações em resina composta. (A) Reconstrução do esmalte palatino com incremento de resina composta na cor WE adaptada e devidamente fotopolimerizada com auxílio da guia de silicone; (B) Incremento de resina composta na cor A2D; (C) Adaptação do incremento de resina composta na cor A1B; (D) Finalização da escultura após o último incremento de resina composta na cor WE. 
Essa matriz foi levada em boca, mantida em posição e fotoativada por 20s. Em seguida, uma camada de resina composta na cor A2D (Forma, Ultradent, Indaiatuba, SP, Brasil) foi adaptada, esculpida e fotoativada (Figura 13B). Posteriormente, para a finalização da restauração estética, camadas de resina nas cores A1B (Filtek Z350 XT, 3M ESPE) (Figura 13C) e WE (Filtek Z350 XT, 3M ESPE) (Figura 13D) foram adaptadas e acomodadas com espátulas e pincel para finalização da escultura.

Ao final, aplicou-se bloqueador de oxigênio (Power Block, BM4, Maringá, Brasil) em todos os dentes restaurados e fotopolimerizou-se por 30s nas faces vestibular e palatina de cada um dos dentes restaurados. Realizou-se acabamento imediato com broca multilaminada de 30 lâminas cilíndrica (FG, Jet Beavers, Ontário, Canadá), lâmina de bisturi no12 para remoção dos excessos cervicais e proximais, disco Sof-Lex de granulação grossa (Sof- Lex Pop On - 3M ESPE, St. Paul, MN, Estados Unidos) para definição das ameias incisais e das áreas de sombra e espelho. Na região proximal, as tiras de lixa abrasiva de granulações média e fina (3M ESPE, São Paulo, Brasil) foram utilizadas para refinar a lisura superficial da região. Por fim, ajustou-se a oclusão com papel carbono em movimentos cêntricos e excêntricos.

Uma semana após o término das restaurações, realizou-se o acabamento e polimento finais. Procedeu-se com a utilização de discos de lixa de granulações grossa e média (Sof- Lex Pop On - 3M ESPE), texturização com broca multilaminada de 30 lâminas cilíndrica ( $F G$, Jet Beavers), borracha abrasiva siliconada grossa (Jiffy Cups, Ultradent Products, Inc., South Jordan, UT, Estados Unidos), discos de lixa de granulação fina e superfina (Sof- Lex Pop On - 3M ESPE) e, por fim, disco de feltro FlexiBuff (Cosmedent, Chicago, IL, Estados Unidos) associado a pasta para polimento de resina Enamelize (Cosmedent, Chicago, IL, Estados Unidos), para maior brilho final (Figuras 14 e 15). 
Finalmente, a paciente foi orientada quanto aos cuidados perante a restauração estética e a necessidade de manutenção preventiva anual pelo cirurgião-dentista. Foi possível observar a satisfação da paciente com o resultado estético obtido, a elevação de sua autoestima, cumprindo com o plano de tratamento proposto frente à queixa principal de maneira conservadora e resolutiva (Figuras 16 e 17).

\section{Discussão}

As anomalias dentárias são um dos fatores que influenciam diretamente na desarmonia do sorriso. A presença de dentes conóides, uma alteração de forma e tamanho na região anterior da maxila ${ }^{4,5,8,14}$, está fora dos padrões de normalidade por conferir um sorriso atípico e infantilizado em decorrência da presença de diastemas em pacientes adultos ${ }^{4,5}$. No caso apresentado, a

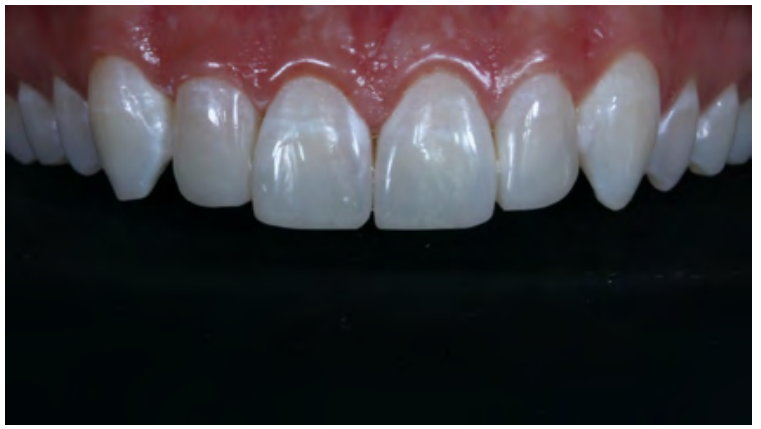

FIGURA 14 - Aspecto final das restaurações em resina composta após acabamento e polimento.

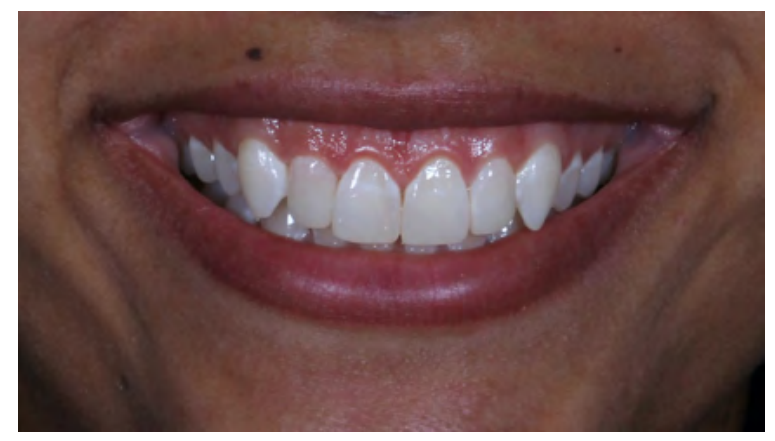

FIGURA 16 - Aspecto final do sorriso sem nenhum desgaste da estrutura dentária.

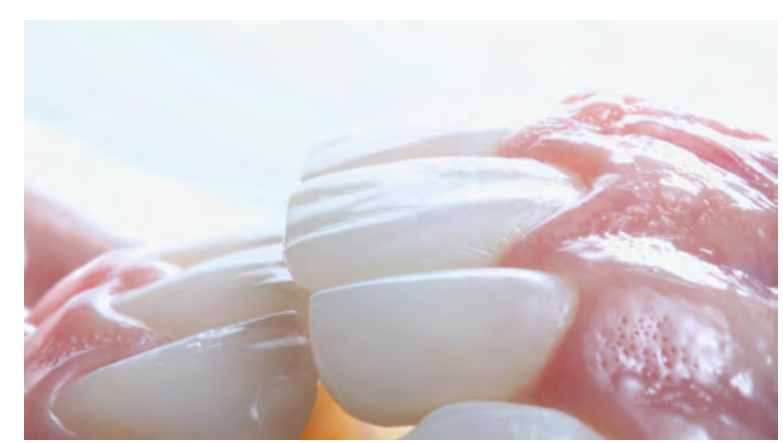

FIGURA 15 - Vista lateral após finalização do acabamento e polimento das restaurações em resina composta. Observe a morfologia, textura e cor dos dentes.

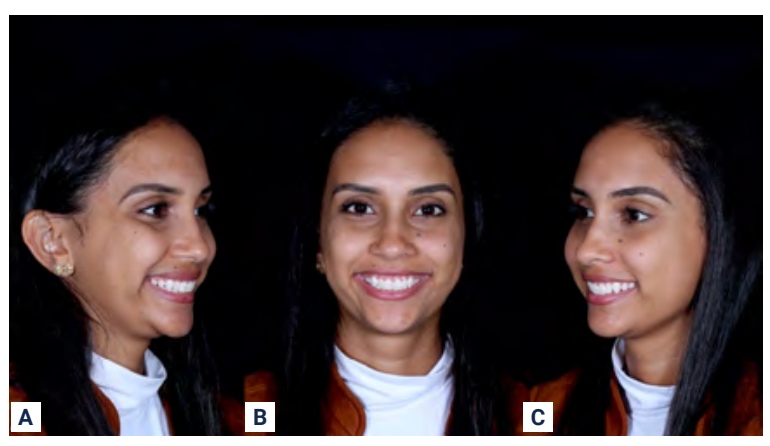

FIGURA 17 - Fotografia da face após o aumento de coroa clínica estético e das restaurações em resina composta. (A) Vista lateral direita do sorriso; (B) Vista frontal; (C) Vista lateral esquerda. 
associação dos dentes conóides com o sorriso gengival aumentava o aspecto infantil do sorriso da paciente. Essa desarmonia ocorre usualmente em incisivos laterais superiores, como neste caso, e para corrigí-la o profissional deve realizar um diagnóstico adequado e um planejamento estético-funcional de acordo com a necessidade e condição financeira do paciente ${ }^{5}$.

A harmonia estética é obtida a partir de um plano de tratamento integrando as diversas especialidades ${ }^{37}$. Diversos procedimentos cirúrgicos periodontais estão disponíveis para fornecer padrões estéticos e o contorno gengival é imprescindível para alcançá-los ${ }^{30}$. A modalidade terapêutica de aumento de coroa clínica estético sem retalho ("flapless") é uma opção minimamente invasiva que dispensa a manobra de síntese ou recobrimento com cimentos cirúrgicos para a finalização do aumento de coroa clínica estético ${ }^{21}$. No caso relatado, optou-se pela técnica de mínima intervenção, pois as características da paciente respeitavam as indicações adequadas: biotipo intermediário e erupção passiva alterada como fator etiológico. Obedecendo rigorosamente a execução da técnica e manutenção da cadeia asséptica no transcirúrgico, a paciente relatou pouco desconforto no pós-operatório e clinicamente observou-se uma reparação tecidual fisiologicamente rápida e eficaz, estando de acordo com os benefícios da técnica ${ }^{21}$.

No planejamento estético reabilitador os critérios dento-faciais, labiais e periodontais devem ser levados em consideração para otimização do sorriso. Em particular, na técnica flapless o estabelecimento da incisão é estipulado pela JCE em dentes naturais sem envolvimento restaurador, ou pela futura margem gengival em dentes já previstos pela reabilitação protética ${ }^{21}$. No caso apresentado, os aspectos periodontais quanto ao contorno, à simetria, ao zênite, à coloração e ao posicionamento gengival foram criteriosamente analisados, e em conjunto, foram determinantes para o posicionamento da incisão, obedecendo ao critério estético, sem a necessidade de envolvimento restaurador no dente por exposição de raiz. 
As modalidades clínicas para resolução dos problemas relacionados com posição, alinhamento, simetria, proporção, forma, textura superficial e cor dos dentes são variadas ${ }^{27}$. Diante da eficácia cientificamente comprovada do clareamento dental, a técnica precedente às restaurações estéticas permite um resultado favorável, visto que aumenta o valor e o brilho do esmalte, acentuando as características de translucidez, também diminuindo a quantidade de desgastes do substrato dentário necessárias para a restauração final ${ }^{2,27-30}$. No presente trabalho, optou-se pelo clareamento caseiro, supervisionado pelo cirurgião-dentista, com peróxido de carbamida $20 \%$, visando não só realizar as restaurações finais com os dentes mais claros, mas minimizar os desconfortos possíveis frente ao agente clareador e potencializar os resultados do tratamento restaurador ${ }^{31,32}$.

O avanço continuado dos materiais dentários e o aprimoramento das técnicas restauradoras tem permitido ao profissional realizar procedimentos minimamente invasivos preservando ao máximo estrutura dentária sadia ${ }^{3,5,7,9,27,30,33}$ com o uso de resinas compostas ou laminados cerâmicos ${ }^{3,9,27,33}$. Estes materiais garantem ao clínico e ao paciente sucesso e longevidade da restauração, quando bem executados ${ }^{9,27}$.

Neste caso optou-se por restaurações diretas em resina composta, devido aos avanços da técnica adesiva e das excelentes propriedades óticas e mecânicas do compósito. Isso tem permitido adicionar e remodelá-las à estrutura dental sem preparo prévio $^{2,3,33}$. Este tipo de restauração possui possibilidade de reparos, custo favorável, otimização do tempo clínico em uma única sessão, reversibilidade, previsibilidade e excelentes resultados estéticos por permitir combinações de cores e efeitos de opacidade, translucidez e opalescência ${ }^{2,4,5,9,30,33,34}$. A longo prazo, no entanto, apresentam coloração instável, maior porosidade, baixa resistência ao desgaste, fraturas e sofrem deformação plástica. Exigem, portanto, habilidade e acuidade do profissional para execução da técnica, além da escolha correta do material 
utilizado, e das consultas de retorno e cuidados do paciente com as mesmas, para que mantenham suas características funcionais e estéticas pelo máximo de tempo possível ${ }^{3,4,9,33}$.

As restaurações adesivas diretas em pacientes jovens também são um desafio clínico para se obter forma, textura e contorno da superfície devido à riqueza de detalhes anatômicos, os quais devem assemelhar-se com a naturalidade da estrutura dentária buscando excelência estética ${ }^{2}$. No caso apresentado, a mimetização dos dentes da paciente foi importante para a obtenção de um resultado o mais natural possível, já que foram restaurados poucos dentes e, nos centrais não foi realizado o recobrimento total da face vestibular dos mesmos com resina composta.

A utilização da matriz ou guia de silicone é um artifício empregado para reconstrução da face palatina das restaurações diretas em resina composta, porém geralmente envolve uma etapa de enceramento diagnóstico que impõe maiores custos e maior tempo clínico. O uso da guia com recorte com broca, como no caso apresentado, otimiza o tempo clínico e simplifica a etapa restauradora, por ser confeccionada diretamente em boca, sob a estrutura a ser restaurada, com as dimensões e contornos planejados de cada elemento sendo definidas imediatamente. A anatomia da face palatina não fica tão bem definida como em casos de guia feita sobre enceramento, mas isso pode ser conseguido com um bom acabamento e polimento após a conclusão das restaurações ${ }^{35}$.

Ademais, o sucesso das restaurações minimamente invasivas depende da escolha correta dos materiais ${ }^{3}$. O uso de resinas compostas nanoparticuladas, como as selecionadas para o caso, é vantajoso, pois apresentam melhores propriedades, quando comparadas às resinas microparticuladas e híbridas, como: boa resistência à fratura e ao desgaste, estabilidade de cor, características ópticas que otimizam a escultura, adequado desempenho físico e biomecânico, textura, brilho e polimento satisfatórios, 
minimização do acúmulo de biofilme, diminuição da contração de polimerização e, sobretudo, boa longevidade ${ }^{36,37}$.

O sucesso clínico das restaurações adesivas compreende o período durante o qual elas são consideradas funcionalmente, biologicamente e esteticamente aceitáveis ${ }^{38}$. As restaurações diretas em resina composta em dentes anteriores possuem em longo prazo uma taxa de insucesso variando de 0 a 4,1\% em acompanhamentos superiores a $3 \operatorname{anos}^{39}$. A necessidade de substituí-las geralmente está relacionada a motivos estéticos, perda de retenção, traumatismo ou hábitos parafuncionais ${ }^{40}$, uma vez que esta técnica é passível de reparos ${ }^{33}$. O cuidado do paciente frente às restaurações mediante hábitos de higiene bucal e dietéticos saudáveis é importante para assegurar a longevidade das mesmas. Sobretudo, os retornos periódicos ao cirurgião-dentista são imprescindíveis para manutenção de polimento, detecção de falhas e eventuais reparos nas restaurações ${ }^{41}$.

\section{Conclusão}

Pode-se concluir que o resultado obtido com aumento de coroa clínica "flapless" e facetas de resina composta melhorou as proporções dento-faciais e gengivais da paciente, solucionando sua queixa principal com preservação de estrutura dental sadia, restabelecendo a estética e mantendo a função de forma adequada. O planejamento individualizado, considerando características de cada caso é indispensável ao sucesso clínico e longevidade de qualquer tratamento proposto, principalmente em pacientes jovens.

\section{Referências}

1 - Alberton SB, Alberton V, Carvalho RV. Providing a harmonious smile with laminate veneers for a patient with peg-shaped lateral incisors. J Conserv Dent. 2017; 20(3): 210-213.

2 - Goyata FR, Costa HV, Marques LHG, Barreiros ID, Lanza CRM, Novaes Júnior JB, Moreno A. Remodelação estética do sorriso com resina composta e clareamento dental em paciente jovem: relato de caso. Archives of Health Investigation. 2017; 6(9): 408-413. 
3 - Reis GR, Vilela ALR, Silva FP, Borges MG, Santos-Filho PCF, Menezes MS. Minimally invasive approach in esthetic dentistry: composite resin versus ceramics veneers. Bioscience Journal. 2017; 33(1): 238-246.

4 - Veronezi MC, Brianezzi LFF, Modena K, Lima MS, Bernardi SE. Remodelação estética de dentes conoides - tratamento multidisciplinar. Revista Digital APO. 2017; 1(1): 35-40.

5 - Sá TLT, Lima LSR, Tuñas ITC. Transformação estética de incisivo lateral conóide com resina composta e auxílio de planejamento digital. Revista Brasileira de Odontologia. 2019; 76: 1-8.

6 - Sousa SJB, Magalhães D, Silva GR, Soares CJ, Soares PFB, SantosFilho, PCF. Cirurgia plástica periodontal para correção de sorriso gengival associada a restaurações em resina composta: Relato de caso clínico. Revista Odontológica do Brasil Central. 2010; 19(51): 362-366.

7 - Campos PRBD, Amaral D, Silva MACD, Barreto SC, Pereira GDDS, Prado MD. Rehabilitation of esthetics in recovering smile harmony: case report. RFO UPF. 2015; 20(2): 227-231.

8 - Kureski PV, Santin GC, Casola HD, Costa JV, Franzin LCS. Incisivos laterais conóides: restabelecendo a harmonia do sorriso. Revista Uningá. 2018; 55(S3): 203-210.

9- Lima MGS, Brito FGB, Araújo Cruz JH, Medeiros LADM, Penha ES, Costa Figueiredo CHM, et al. Reanatomização do sorriso com uso de resina composta: relato de caso. Archives of Health Investigation. 2020; 8(9): 501-505.

10 - Laverty DP, Thomas MBM. The restorative management of microdontia. British Dental Journal. 2016; 221(4): 160-166.

11 - Fekonja, A. Prevalence of dental developmental anomalies of permanent teeth in children and their influence on esthetics. Journal of Esthetic and Restorative Dentistry. 2017; 29(4): 276-283.

12 - Neville B, Bouquot JE, Damm DD, Allen CM. Patologia oral e maxilofacial. 3. ed. Rio de Janeiro: Elsevier; 2009. 82 p.

13 - Pegoraro LF, Valle AL, Pegoraro TA, Corotti KM, Vidotti HA. Resolution of complex esthetic problems in abnormal anterior teeth: a clinical report. The Journal of prosthetic dentistry. 2014; 112(2): 94-98.

14 - Martini AP, Souza FI, Mazza LC, Melo RAC, Araújo NS. Esthetic Treatment of Conoids Lateral Incisor Laminate veneers: A2-Year Follow-Up. Int J Dent Oral Health. 2016; 2(4): 1-4.

15 - Tirone F, Salzano S, Rolando E. Adhesive esthetic treatment of nonsyndromic maxillary anterior microdontia in young high-demanding patients: a case series. Int J Esthet Dent. 2016; 11(4): 520-537.

16 - Chen Y, Zhou F, Peng Y, Chen L, Wang Y. Non-syndromic occurrence of true generalized microdontia with hypodontia: A case report. Medicine (Baltimore). 2019; 98(26): e16283. 
17 - Pires CV, Souza CGLG, Menezes SAF. Procedimentos plásticos periodontais em paciente com sorriso gengival: relato de caso. $\mathrm{R}$ Periodontia. 2010; 20(1): 48-53.

18 - Diaspro A, Cavallini M, Piersini P, Sito G. Gummy Smile Treatment: Proposal for a Novel Corrective Technique and a Review of the Literature. Aesthet Surg J. 2018; 38(12): 1330-1338.

19 - Pedron IG, Mangano A. Gummy Smile Correction Using Botulinum Toxin With Respective Gingival Surgery. J Dent (Shiraz). 2018; 19(3): 248-252.

20 - Bhola M, Fairbairn PJ, Kolhatkar S, Chu SJ, Morris T, Campos M. LipStaT: the lip stabilization technique- indications and guidelines for case selection and classification of excessive gingival display. Int J Periodontics Restorative Dent. 2015; 35(4): 549-559.

21 - Carvalho PFMD, Silva RCD, Joly JC. Aumento de coroa clínica estético sem retalho: uma nova alternativa terapêutica. Rev. Assoc. Paul. Cir. Dent. 2010; 64(1): 26-33. (Edição especial)

22 - Nasr MW, Jabbour SF, Sidaoui JA, Haber RN, Kechichian EG. Toxina botulínica para o tratamento da exposição gengival excessiva: uma revisão sistemática. Aesthet Surg J. 2016; 36(1): 82-88.

23 - Dym H, Pierre R. Diagnosis and Treatment Approaches to a "Gummy Smile”. Dent Clin North Am. 2020; 64(2): 341-349.

24 - Silva DB, Zaffalon GT, Corazza PF, Bacci JE, Steiner-Oliveira C, Magalhães JCA. Cirurgia plástica periodontal para otimização da harmonia dentária relacionada ao caso clínico. Revista Brasileira de Saúde. 2010; 1(1): 31-36.

25 - Malamed SF. Manual de anestesia local. 6th ed. Rio de Janeiro: Elsevier; 2013. 146 p.

26 - Gargiulo AW, Wentz FM, Orban B. Dimensions and relations of the dentogingival junction in humans. The Journal of Periodontology. 1961; 32(3): 261-267.

27 - Bandéca MC, Calixto LR, Pinto SCDS, Clavijo VRG, Tonetto MR, Oliveira Júnior OB, et al. Clareamento e restauração adesiva direta para correção de desarmonias estéticas. International Journal of Brazilian Dentistry. 2010; 6(3): 324-334.

28 - Wittmann K, Giacomelli Neto R, Longo RE, Sene F. Restauração estética do sorriso por meio da integração de técnicas de clareamento e restauração direta de resina composta. International Journal of Brazilian Dentistry. 2013; 9(4): 454-465.

29 - Vieira-Dantas ED, Cavalcanti YW, Carvalho WLD, Assunção IVD, Santos AJSD. Clareamento dentário como etapa prévia à restauração de dentes com alteração severa de cor. Revista Brasileira de Ciências da Saúde. 2014; 18(1): 41-48. 
30 - Cunha LF, Gaião U, Silva RC, Gonzaga CC, Correr GM. Cosmetic remodeling of the smile: combining composite resin and ceramics over teeth and implants. Case reports in dentistry. 2017; 2017: 1-7.

31 - Santana GO, Silva MJA. Clareamento dental e a influência da dieta na estabilidade da cor: revisão de literatura. Mult. Psic. 2019; 13(48): 268279.

32 - Qin J, Zeng L, Min W, Tan L, Lv R, Chen Y. A bio-safety toothwhitening composite gels with novel phthalimide peroxy caproic acid. Composites Communications. 2019; 13: 107-111.

33 - Carrijo DJ, Ferreira JLF, Santiago FL. Restaurações estéticas anteriores diretas e indiretas: revisão de literatura. Revista Uningá. 2019; 56(S5): $1-11$.

34 - Almeida ES, Rocha BB, Carvalho FR, Leão PCN, Andrade MJ. Odontologia minimamente invasiva, uma análise sobre facetas cerâmicas: revisão de literatura. Rev Mult Psic. 2019; 13(47): 940-952.

35 - Bertholdo G, Albino LGB, Ricci WA. Matriz Bertholdo/Ricci/Barrotte (BRB): uma simplificação de técnica para obtenção de guia de estratificação com compósitos. Clínica. 2014; 10(2): 204-13.

36 - Pereira DA, Borges MG, Silva FP, Menezes MS. Reabilitação estética do sorriso por meio de procedimento restaurador direto com resina composta nanoparticulada: relato de caso. Revista Odontológica do Brasil Central. 2016; 25(72): 54-58.

37 - Velo MMDAC, Coelho LVBF, Basting RT, Amaral FLBD, França FMG. Longevity of restorations in direct composite resin: Literature review. RGO-Revista Gaúcha de Odontologia. 2016; 64(3): 320-326.

38 - Dietschi D, Shahidi C, Krejci I. Clinical performance of direct anterior composite restorations: a systematic literature review and critical appraisal. The international journal of esthetic dentistry. 2019; 14(3): 252-270.

39 - Demarco FF, Collares K, Coelho-de-Souza FH, et al. Restaurações compostas anteriores: uma revisão sistemática sobre sobrevida a longo prazo e razões para falha. Dent Mater. 2015; 31(10): 1214-1224.

40 - Collares K, Opdam NJM, Laske M, et al. Longevity of Anterior Composite Restorations in a General Dental Practice-Based Network. J Dent Res. 2017; 96(10): 1092-1099.

41 - Beck F, Lettner S, Graf A, et al. Survival of direct resin restorations in posterior teeth within a 19-year period (1996-2015): a meta-analysis of prospective studies. Dent Mater. 2015; 31(8): 958-985. 


\title{
Esthetic restoration of conoid teeth with gingival contouring in a young patient: a case report
}

\begin{abstract}
The search for dental treatments for aesthetic reasons has increased a lot due to the great impact of the smile on the self-esteem and well-being of patients in their social life. In esthetic rehabilitation planning, dento-facial, labial, periodontal and personal criteria must be taken into account when indicating any treatment. The purpose of this paper is to report a case of gingival smile and conoid teeth in a young patient, treated with a minimally invasive approach. On physical examination, it was possible to observe the gingival smile caused by the altered passive eruption and isolated microdontia on the upper lateral incisors, which impaired the harmony of the smile. Some treatment plans have been proposed and in view of the cost-benefit, an esthetic crown lengthening ona flapless technique of both central and lateral incisors and on premolars of the upper right side was performed. Associated with supervised home tooth whitening and direct veneers in composite resin without prior preparation of the dental structure. The restorations were guided by a silicone matrix obtained immediately and worn to the final size planned with a drill, without the need for diagnostic waxing. The result obtained with the proposed treatment improved the dento-facial and gingival proportions, solving the patient's main complaint with preservation of a healthy dental structure, improving the esthetic and maintaining a proper function.
\end{abstract}

KEYWORDS: Dental Esthetics; Composite Resins; Periodontics; Plastic Surgery. 